CLINICAL ETHICS

\title{
Informed consent in the Pakistani milieu: the physician's perspective
}

\author{
A M Jafarey, A Farooqui
}

J Med Ethics 2005;31:93-96. doi: 10.1136/jme.2002.002220

Informed consent enjoys an unassailable position in both clinical and research situations as a safeguard of patients' rights. Keeping the patient involved in the decision making process is easier when there is direct communication with the individual. The Pakistani milieu offers challenges to this process because crucial decision making is often done by family members or is left entirely up to the attending physician. There seems to be a general acceptance of this shifting of focus from the individual to other players. This also raises certain ethical dilemmas for physicians who may feel uncomfortable with communication which excludes the patient or in accepting a paternalistic primary decision making role. The objective of this informal qualitative study was to ascertain physicians' perceptions regarding the process of information delivery to the patient in the Pakistani context and the various influences acting upon it.

See end of article for authors' affiliations

\section{Correspondence to:} Dr A M Jafarey, Department of Surgery, Aga Khan University, PO Box 3500, Stadium Road, Karachi, 74800, Pakistan; jafarey@akunet.org; aamir.jafarey@aku.edu

Received 21 October 2002 In revised form 3 November 2003 Accepted for publication 4 December 2003
$\mathrm{T}$ - he stipulation that an individual be fully conversant with all the relevant facts is central to the concept of informed voluntary consent both in research and clinical situations. ${ }^{1}$ Only then can the process of informed consent play the role of a safeguard that upholds autonomy and acknowledges the fundamental position of the individual's choice. ${ }^{2}$ Centrality of the individual in the medical decision making process is sacrosanct in the West with the patient actively involved in all discussions with the physicians. ${ }^{3}$ The importance of individual autonomy however is challenged in many Eastern cultures. ${ }^{4}$ In the Pakistani context, there are several other factors to consider in crucial decision making situations. In some cases, the patient opts to exclude himself from receiving information and participating in discussions regarding his management, delegating family members to make the decisions. Alternatively he may invite his physician to use his best judgment and choose the best option for him. In other situations, the family members may insist on excluding the patient from the decision making loop. This family, physician, and patient triad in Pakistani society in medical decision making has been comprehensively documented by Moazam. ${ }^{5}$ A tradition of living in extended families, often with shared incomes, strengthens the role of the family in major decision making situations like selection of marriage partners, financial transactions, and in making decisions about medical treatment.
Requests from patients like "Doctor Sahib, you are like my elder sibling or parent. You know what is best-you decide for me" are not uncommon in this part of the world. How should the physician proceed when the onus of decision making is thrust upon his shoulders? Should he accept the imposed paternalistic role and proceed according to his own best judgment, respecting the patient's right to exercise autonomy and decision not to know? Or should he still insist on involving the patient? Is the physician, in performing his "duty" to convey information to the patient-irrespective of the patient's desireactually respecting the patient's autonomy?

In another common scenario, a family member may take the physician aside and ask him to carry on with his management but not to tell the patient that she has a life threatening disease as she is "faint of heart" or would not be able to "sustain the shock". Here again the physician faces the conflict between upholding the patient's right to know and respecting the desire of the family to shield the patient from bad news.

This change of focus from the individual to the family or the physician raises the question of the role of individual autonomy in this culture and the whole concept of informed consent. Who needs to be informed and who will participate in making the decisions? The apparently different set of ground rules gives rise to numerous ethically challenging situations in clinical practice in Pakistan.

There is paucity of information regarding the various factors affecting the process of information delivery and medical decision making in Pakistan and the importance of the different key players influencing the process. This informal qualitative study was designed to gain deeper insight into physicians' perspectives regarding this process. The objective was to evaluate how physicians at this institution approach such situations in their clinical practice. What are their guiding principles in the process of informing the patient about his disease, its prognosis, and treatment? We also explored the impediments they face in the process and ascertained the level of satisfaction with their practice in this regard.

\section{METHODS}

This informal qualitative study was conducted at Aga Khan University Hospital, Karachi, Pakistan, a private sector tertiary care hospital. Three approaches were used which included focus group discussions, individual in-depth interviews, and informal discussions to gather data. The perceptions about informed consent from 
various levels of physicians including professors, associate professors, assistant professors, senior instructors, fellows, and chief residents were obtained. These physicians were from specialties including surgery and allied fields, gastroenterology, oncology, rheumatology, family medicine, cardiology, neurology, and invasive radiology.

The commonality among the various specialties listed above was based on their interaction with patients and frequent need for passage of information from them to the patients. Their diversity lies in the different kinds of diseases they deal and the various modes of interventional and noninterventional treatments they apply in the dispensation of healthcare.

A facilitator introduced the topic to the participants of the focus group discussion and put forward non-leading questions to highlight the issue being discussed. In each focus group 10-12 physicians from various specialties participated in the discussion. In order not to influence the proceedings, the facilitator did not participate in the discussion. Comments were recorded and simultaneous notes were also taken. These were immediately transcribed and authenticated by both the authors. The transcribed material was then analysed for identifying the various responses from each group and then grouped accordingly in the result. We stopped after the fifth focus group discussion because of overlapping information.

Seven key informants who had not participated in the focus group discussions or the informal discussions were identified from major specialties. Verbal consent was taken from each participant. The facilitator used the informal free conversational approach for the interviews, the main aim being to obtain personal experiences that would further expand on the perspective. These interviews were transcribed immediately after the meeting and the transcriptions individually analysed for identification of the key points.

Five informal interviews were carried out on the basis of convenience sampling. Physicians who were available for the interview were requested to participate in the exercise in an informal manner. A verbal consent was also obtained from them before the interview. These interviews were also transcribed immediately after the meeting. Again the transcriptions from each individual were analysed for key points.

\section{RESULTS}

The discussions were focused in the area of information delivery in the process of informed consent and the roles played by the various key participants. Apprehensions regarding the various pitfalls inherent to this process in our society were also debated. The physicians also talked about the extent of their moral satisfaction with the process of information transfer as practiced at present and suggestions for improvement were put forward, keeping in mind cultural and social values.

\section{Information}

Most participants felt that it was the physician's duty to bring the patient into the decision making process. The prevailing opinion across the different focus groups was that regardless of the opinion of the patient or the family, the physician is obligated to deliver at least some basic information to the patient. It was a general opinion that information about the disease and the treatment ensures participation and helps the patient respond better to the management. The patient could therefore not absolve himself from his right to know what was happening to him and nor could the family take away this right from him.

There was wide divergence of view regarding the extent of information to be provided, from giving encyclopaedic levels of information to outright deception. The majority felt that the process needs to be individualised, and a tiered approach was suggested, starting from the basic facts and going further based on the patient's demands. Some participants emphasised outlining the risks versus the benefits of a particular therapy to the patient. There was no consensus on how to identify essential information from the "details" that could be omitted.

The issue of voluntarily withholding information came up repeatedly in the discussions. Most participants agreed that it was perfectly acceptable to use alternative words like "growth" or "mass" rather than use the term "cancer" and this did not amount to deception. The general view was that the patients usually already knew or suspected strongly what was wrong with them, especially patients with malignancies.

Some of the physicians were comfortable with entirely excluding the "more distressing" facts about their disease or giving a more "optimistic" picture to the patient. Physicians as opposed to surgeons were more comfortable in withholding the exact diagnosis if they felt that revealing all the facts could enhance the distress of the patient and not contribute meaningfully towards the management. "You can be evasive regarding the diagnosis if the patient does not ask you directly" said one physician. This group however was of the firm opinion that if a procedure has to be undertaken, it has to be explained to the patient even if the family says otherwise. This was necessary to ensure the patients cooperation for the procedure.

\section{Role of the family}

When a family member is taken ill, it is common to see several family members accompanying the patient to the doctors' office and if a patient is admitted for treatment, one can often see several family members camping outside in the open for days. "Not only does the patient bring his family along when he comes to visit the doctor, at times it seems he has brought along the entire family tree!" remarked one participant.

\section{"There is no difference between the patient as an individual and his family. Both are one and the same."}

All participants accepted the key position enjoyed by the family in decision making processes in Pakistan. Many of the participants refused to draw a distinction between the patient and the family in this culture, claiming that they are inseparable and should be addressed as a one unit. "There is no difference between the patient as an individual and his family. Both are one and the same" said one physician. Several participants pointed out that the doctor first confronts the family members with the diagnosis, especially when suspecting cancer, before approaching the patient. Often a joint strategy is hurriedly arrived at between the physician and the family before approaching the patient. The question asked by many was "how then can the role of the family be relegated to anywhere below that of the patient himself?"

\section{Apprehensions}

Several participants voiced their apprehensions in following a Western oriented blind information delivery policy without considering the wishes of the patient and the family. One fear of spelling out details of the diagnosis, the prognosis, and possible complications of procedures was of scaring the patients away to other practitioners with a more reassuring "trust me, you will be fine" approach. Losing patients translates into losing valuable experience and revenue and is a tightrope that needs to be walked at times. As one relatively junior surgeon said, "I try and tell the patient all 
possible complications of a procedure so that if something does go wrong, at least he was forewarned. I however tend to loose patients also by this approach as they sometimes choose to go to a surgeon who does not alarm them with all the possibilities". This candidly expressed fear of losing patients by providing too honest an informed consent may tempt physicians to "recruit" patients by giving a rosy "nothing will go wrong" picture. This appeared to be more of an issue for the relatively junior staff still in the formative stages of their practices and it came up several times in the discussions.

A concern that was voiced repeatedly was whether we as physicians were imposing "foreign" values on the patients by thrusting upon them unwanted and unsolicited autonomy. Participants felt that they were unclear about the importance and the relevance of autonomy for the end user-the patient-and in dragging them into the decision making process we may actually be harming them in an unintended way. "The job of a doctor is to reassure and comfort the sick, not to frighten them" said one surgeon, quoting a patient.

Although physicians were generally willing to let the physician-patient-family balance remain undisturbed, a concern was voiced regarding the legality of making decisions in consultation with the family even if it was done at the insistence of the patient himself. It was pointed out that in the event of a complication there was nothing stopping the patient from turning around and saying that he was not made aware of the choices before the operation and then proceeding with litigation.

Some participants felt that there was no mechanism in place to capture the dialogue between all concerned parties that went into the process of informing the patient, assessing his understanding, and getting his permission.

\section{Factors adversely affecting the process}

Several factors came across as having an adverse effect on the process of informed consent. One such issue was time: the process of obtaining a truly informed and understood consent requires time and patience, both deficient in busy clinical practices. A tiered process (as advocated above) requires that the understanding of the patient be gradually built up and comprehension verified to an extent that satisfies both parties. In busy practices this becomes more and more difficult. As one surgeon said, "I am quite satisfied with my interaction with the patient regarding the informed consent process right now but I am not sure if this would remain so as my practice gets busier".

One fact that kept coming up was the influence of the level of intelligence of the patient on the quality of the interaction. There seemed to be an obvious hesitation on the part of the participants in equating the lack of intelligence with an inability to comprehend what was being told. Participants were however much more comfortable in saying that a higher level of intelligence did facilitate communication. It was emphatically added that a perceived lack of intelligence should not be a reason not to communicate. A common statement was "every effort should be made to come down to the level of the patient in order to explain the facts".

Another area that raised forceful objections was in equating education with intelligence. Participants were generally of the view that both could not be equated and an uneducated person could be intelligent and vice versa. But a lack of education was also pointed out as a hindrance in satisfactory communication and again participants emphasised the need for more effort to get the message across to an uneducated patient.

A physician pointed out that the female sex carries with it its own issues in this society. In obstetrics practice for instance, women may be unwilling to acknowledge any information given to them or sign a consent document unless they have their husbands present. They may even be happier leaving the discussion entirely to their spouses, willingly assuming a back seat in the decision making process concerning their own health.

\section{"I take ten minutes to tell the interpreter what is wrong with the patient and he takes inside of a minute to talk to the patient and obtain the thumb imprint."}

Another important factor for the hospital at which this study was conducted was that of consent through interpreters. Afghan refugees with no knowledge of the local languages or English constitute a significant proportion of patients in this hospital. Apart from one surgeon who had learnt enough Persian to get by, all other doctors used the volunteer interpreter service. None of the physicians were entirely satisfied with the use of the non-professional interpreters and, although they were fulfilling the legality by obtaining the signature or thumb imprint on the dotted line, they were dissatisfied by the quality of information delivered. "I take ten minutes to tell the interpreter what is wrong with the patient and he takes inside of a minute to talk to the patient and obtain the thumb imprint" said one doctor. Physicians had devised ways of getting around this problem like making the interpreter translate one sentence at a time to make sure all the facts were delivered. All voiced a desire to have a professional service of trained interpreters who know the importance of conveying the information correctly, checking comprehension and then carrying out the legal formalities.

\section{Satisfaction with the process}

The level of satisfaction of the physicians with the information delivery process depended primarily upon the achievement of two criteria: delivering information to the patient and assessing comprehension. Those physicians whose practices allowed them to spend more time with the patients were more satisfied than those with busier practices. Experience in dealing with a variety of patients and families also made a difference.

One factor that helped physicians do the job better was interaction with a well informed patient. Cardiac patients for instance, referred to a cardiologist for angiographies, were found to be generally well informed of their disease and the various options available. It was therefore easier to communicate with them and satisfactorily agree on a plan. It was stressed that publicity or educational material like brochures describing a procedure also appeared to facilitate the process for the physicians.

Physicians who do a specific variety of invasive procedures such as gastroenterologists and cardiologists, invasive radiologists, or physicians who see only a certain variety of patients, like oncology patients, were generally more satisfied by the process of informed consent. They had devised their own mental checklists of "must tell" points to the patients and as long as they used them, they were content. In fact, the only person "absolutely" satisfied by his practice of informing the patients of their disease, its prognosis, and the plan of therapy was an oncologist: "There are six things that a patient must know before commencing chemotherapy. I make sure they understand them and then I proceed." Some physicians advocated the development of checklist type consent forms for major procedures in all fields. Even if it meant the form to be spread over several pages, all the important agreed upon points would be covered. The participants most unsatisfied with the process of information delivery were the surgeons. Perhaps a wider variety of pathology that they see prevents them from devising these mental pathways for each patient. 


\section{COMMENTS AND CONCLUSIONS}

The consent process has two distinct but not necessarily incompatible objectives. It acts as a tool to minimise chances of legal action resulting from a complication of therapy by providing prior information regarding the possible eventualities and getting the patient's agreement before proceeding further. It also emphasises the moral responsibility of the physician in acknowledging the autonomy of the patient and ensuring his inclusion in the decision making process. Completing one component does not necessarily imply that the requirements for the other have also been fulfilled. ${ }^{7}$ These sentiments were echoed by the participants in our study who felt that the consent form was a mere legality and its completion did not signify the conclusion of the moral responsibility of the physician in keeping the patient informed.

The recommended method for obtaining an informed consent in this hospital requires the consultant to have a detailed discussion regarding the various aspects of the recommended procedure with the patient at the time of booking for the procedure. The patient is asked to sign a printed consent form available in Urdu and English if he agrees with the plan. This exchange usually takes place in the out patients' clinic before admission for the procedure. Although the paperwork is taken care of, the patient obviously has the right to retract consent at any time. This delay from the time of signing the consent until the actual procedure enables the patient to have sufficient time to discuss and reflect on his decision, and he has the option of contacting the physician again for further clarification.

There was unanimity regarding the importance of delivering information to the patient regarding major aspects of the treatment and bringing him into the decision making loop. There has been an increasing trend over the past three or four decades towards more disclosure, even in cultures where physician paternalism is traditionally well accepted like Japan, and Eastern and Southern Europe. ${ }^{8}$ Even in the US where personal autonomy has always been of overriding importance, physicians were much less likely to disclose the diagnosis of cancer to their patients in the early 1960s than they were by the end of the seventies. ${ }^{9}$

There was considerable lack of clarity regarding the extent of information considered adequate. Some physicians felt there was a place for evasiveness as far as disclosure of the diagnosis was concerned if it protected the patient from additional distress. The respect enjoyed by the physician in our society imposes certain moral responsibilities on them, one being the balanced presentation of facts which neither unduly alarm nor entice patients but facilitate decision making.

The quality of consent was clearly equated with the amount of time spent and the experience of the physician. Most physicians felt that lack of intelligence or illiteracy influenced comprehension negatively but could be overcome by spending more time in imparting information. Following checklists was found by some physicians to be an efficient way of delivering information, especially for routine procedures, and there were suggestions to consider devising checklist type of consent documents for all commonly performed procedures.

Most physicians also acknowledge the family and the patient as inseparable entities in this society and found it morally acceptable to include the family in the decision making process along with the patient. "In Pakistan, for the vast majority of the population, you are your family, and the family is you. ${ }^{\prime 5}$ One way of officially endorsing the role of the family in the informed consent process is to identify a next of kin formally on the consent form as a legal representative of the patient. This could remove inhibitions that some physicians experienced in communicating "through" a family member rather than with the patient directly. With a legally identified and duly authorised next of kin the fear of litigation would also be eliminated.

An apprehension repeatedly surfacing was that physicians were basing practices on what they personally considered to be appropriate values. In the words of one surgeon, "The physician has the best interest of the patient in mind and it is in the best interest of the patient to know about the disease." This paternalistic sentiment was echoed by others also. This viewpoint may not necessarily be shared by the patients. Are we attempting to respect the patient's autonomy without actually knowing whether the patient understands the autonomy that we are insisting on upholding?

This study has brought out several areas of concern in the moral aspects of the existing informed consent process and a few practical suggestions have also emerged. A lacuna in our understanding that has been identified is in the area of patients' values regarding autonomy and their opinion concerning the role of the various players in the process of informed consent. Another area that remained untouched in the discussions undertaken for this study is the teachings of Islam regarding individual rights and caregivers' responsibilities.

Any attempt to gain a deeper insight into the role of the individual, family, and the physician in medical decision making in the Pakistani context will remain incomplete without going into the teachings of Islam in this respect. It is also imperative that the patient's opinions regarding the role of the various influences acting upon the process are also taken into account so that policies and practices can be based on locally acceptable facts and not on imported ideals. These could be areas of further research in order to unravel the patient, family, and physician triad in the Pakistani society.

\section{Authors' affiliations}

A M Jafarey, A Farooqui, Department of Surgery, Aga Khan University, Karachi, Pakistan

\section{REFERENCES}

1 Beauchamp TL, Childress JF. The Principles of Biomedical Ethics, 4th edition. New York: Oxford University Press, 2001.

2 Joseph AC, Lorna AR. Western bioethics on the Navajo reservation: benefit or harm? JAMA 1995;274:826-9.

3 Leslie JB, Sheila TM, Gelya F, et al. Ethnicity and attitudes toward patient autonomy. JAMA 1995;274:820-5

4 Kerry WB, Edwin CH. Bioethics for clinicians: 20. Chinese bioethics. Can Med Assoc J 2000; 163:1481-5.

5 Moazam F. Family, patient and physician in medical decision making: a Pakistani perspective. Hastings Cent Rep 2000;6:28-37.

6 Correia JC. Autonomy and identity. J Med Ethics 2000;26:141.

7 Lantos J. Informed Consent. The whole truth for the patient? Cancer 1993;72(Suppl 9):2811-15.

8 Horikawa N, Yamazaki T, Sagawa M, et al. Changes in disclosure of information to cancer patients in a general hospital in Japan. Gen Hosp Psychiatry 2000;22:37-42

9 Novach DH, Plumer R, Smith RL, et al. Changes in physicians attitudes toward telling the cancer patient. JAMA, 241:897-900. 